\title{
Two-channel Snapshot Mueller Matrix Polarimeter
}

\author{
Matthieu Dubreuil $^{1,2}$, Sylvain Rivet ${ }^{1,2}$, Bernard Le Jeune ${ }^{1,2}$ and Jack Cariou ${ }^{1,2}$ \\ 1) Université Européenne de Bretagne, France
}

2) Université de Brest, Laboratoire de Spectrométrie et Optique Laser (E.A. 938), 6 Avenue le Gorgeu, C.S. 93837, 29238 Brest Cedex, France

*Corresponding author: matthieu.dubreuil@univ-brest.fr

This paper describes a new set-up for a Snapshot Mueller Matrix Polarimeter (SMMP). It relies on the separation and orthogonal polarization of two light beams by a Wollaston prism located at the set-up output. The simultaneous treatment of the two spectra allows an enhancement of accuracy for real-time measurements through reduction of the effects caused by random noise and systematic errors. Moreover, it gives insight into the non-uniform spectral response of the medium under study. Experimental results support the feasibility of the proposed technique. (c) 2009 Optical Society of America.

OCIS codes: (230.5440) Polarization-selective devices, (260.5430) Polarization.

\section{1- Introduction}

Mueller matrix polarimetry is a powerful method for optical characterization of samples. It is currently used in various scientific fields such as ellipsometry [1], rheology [2] or medical diagnosis [3]. Many Mueller matrix polarimeters have been implemented over the last two 
decades. Most of them make fast measurements $(\sim s)$ of the polarimetric response of a sample under study [4-6]. However, a temporal monitoring of a Mueller matrix, [M(t)], at different time scales thanks to ultrafast measurements $(<m s)$ would widen the scope of Mueller polarimetry. Encoding the polarization states with the wavelength so as to carry out polarimetric measurements with an acquisition time that only depends on the detection system aperture was proposed in $[7,8]$. A Snapshot Mueller Matrix Polarimeter (SMMP) by wavelength polarization coding was recently developed within our laboratory $[9,10]$. This instrument makes instantaneous measurements of the polarimetric response of a sample. Evidence of the experimental feasibility of the technique was provided in [9], and the specific systematic errors liable to appear with this new kind of polarimeter were highlighted in [10]. However, the quality of measurement with an SMMP can be impaired by random noise, systematic errors associated to the retarder plates and possible interference effects due to multiple reflections by the medium under study. This paper describes a set-up derived from an SMMP and named two-channelSMMP. The improvements generated by the new configuration are studied through: i) a reduction of the impact by random noise or systematic errors and ii) a correction of the nonuniform response by the sample (interference effects) in absorbing and non-absorbing media. These improvements are highlighted by experimental results.

\section{2- Presentation of the device}

Figure 1 presents this new set-up derived from the single-channel-SMMP described in [9]. The light is issued from a spectrally broadband illumination system, and its polarization is encoded by an input linear polarizer and two birefringent wave-plates of thickness, $e$. After interaction with the medium, the polarized-light is decoded with two birefringent wave-plates of thickness, $5 e$, and an output Wollaston prism. The only difference with the single-channel- 
SMMP described in [9] is the use of a Wollaston prism instead of an output linear polarizer. Thus, the two output channels, which are spatially separated and orthogonally polarized, are focused on two optical fibers linked to a spectrometer (grating and CCD array). Two intensity spectra, denoted by $I^{\perp}(\lambda)$ and $I^{\prime \prime}(\lambda)$, are measured simultaneously and correspond to the two orthogonally-polarized light beams issued from the Wollaston prism. The expression of the detected intensities can be derived from the Mueller formalism. To evaluate the effect of the setup polarizing-elements on an input Stokes vector, $\vec{S}_{i}$, let us denote by [M] the Mueller matrix of an unknown sample; moreover, $\left[P_{o l}(\theta)\right]$ is the Mueller matrix of a linear polarizer with an azimuth angle, $\theta$, and $[B(\phi, \theta)]$ is the Mueller matrix of a linear birefringent wave-plate whose retardation and fast axis orientation are $\phi$ and $\theta$, respectively. The intensities are:

$I^{\perp}(\lambda)=\left[\begin{array}{llll}1 & 0 & 0 & 0\end{array}\right]^{T}\left[P_{o l}(\pi / 2)\right] \cdot[B(5 \phi, \pi / 4)] \cdot[B(5 \phi, 0)] \cdot[M] \cdot[B(\phi, 0)] \cdot[B(\phi, \pi / 4)] \cdot\left[P_{o l}(0)\right] \cdot \vec{S}_{i}$

$I^{\prime \prime}(\lambda)=\left[\begin{array}{llll}1 & 0 & 0 & 0\end{array}\right]^{T}\left[P_{o l}(0)\right] \cdot[B(5 \phi, \pi / 4)] \cdot[B(5 \phi, 0)] \cdot[M] \cdot[B(\phi, 0)] \cdot[B(\phi, \pi / 4)] \cdot\left[P_{o l}(0)\right] \cdot \vec{S}_{i}$,

where the symbol ${ }^{T}$ represents the transpose operator of a column vector. In the first-order approximation, the retardation of a linear birefringent wave-plate (birefringence, $\Delta n$, and thickness, $e$ ), can be expressed as $\phi=\phi_{0}+f_{0} \lambda$, where $\phi_{0}$ is the first-order retardation: $\phi_{0}=(2 \pi \Delta n e) / \lambda_{0}$, and $f_{0}$ is the fundamental frequency associated to the reference thickness $(e)$. The signals $I^{\perp}(\lambda)$ and $I^{\prime \prime}(\lambda)$ are both periodic and can be rewritten as follows:

$$
I^{\perp}(\lambda)=s(\lambda) \cdot \operatorname{Re}\left[g_{0}^{\perp}+\sum_{n=1}^{12}\left(g_{n}^{\perp}+i h_{n}^{\perp}\right) \cdot e^{i\left(n f_{0} \lambda+\phi_{w}\right)}\right]
$$




$$
I^{\prime \prime}(\lambda)=s(\lambda) \cdot \operatorname{Re}\left[g_{0}^{\prime \prime}+\sum_{n=1}^{12}\left(g_{n}^{\prime \prime}+i h_{n}^{\prime \prime}\right) \cdot e^{i\left(n f_{0} \lambda+\phi_{w}\right)}\right]
$$

where $s(\lambda)$ contains the source spectrum shape and, possibly, the non-uniform spectral response of the medium under study; moreover, $g^{\prime \prime}{ }_{n}$ and $h^{\prime \prime}{ }_{n}$ are both linear combinations of the Mueller coefficients, $m_{i j}$, in the parallel case, whereas $g^{\perp}{ }_{n}$ and $h^{\perp}$ are those in the perpendicular one. As all of the Mueller coefficients are assumed to be wavelength-independent, the analysis is made on a narrow $(10 \mathrm{~nm})$ spectral detection range. The signal is composed of 13 frequencies (from 0 to $12 f_{0}$ ), and the phase, $\phi_{w}$, results from the windowing of the measured signal during the analysis (Blackman window). A more detailed discussion of this variable is available in [10].

\section{3- Random noise}

As the Mueller matrix reconstruction is performed in the Fourier domain, the magnitudes of the Fourier peaks in the real part and those in the imaginary part are, respectively, $g^{\prime \prime}{ }_{n}\left(g^{\perp}{ }_{n}\right)$ and $h^{\prime \prime}{ }_{n}\left(h^{\perp}\right)$ in the parallel (perpendicular) case. The measurement of the magnitudes of the Fourier peaks issued from a single spectrum allows one to retrieve the 16 Mueller coefficients of a sample. Expression of the relationships between the Fourier peaks and the Mueller coefficients in a matrix formalism leads to $\mathbf{v}=\mathbf{P} . \mathbf{m}$, where $\mathbf{v}$ is a 50 -dimension vector whose components are the magnitudes of the Fourier peaks, $\mathbf{v}=\left[g^{\prime \prime}{ }_{0}, \ldots, g^{\prime \prime}{ }_{12}, h^{\prime \prime}{ }_{1}, \ldots, h^{\prime \prime}{ }_{12}, g^{\perp}{ }_{0}, \ldots, g^{\perp}{ }_{12}, h^{\perp}{ }_{1}, \ldots, h^{\perp}{ }_{12}\right]^{T}$, and

$\mathbf{m}$ is a 16-dimension vector composed of the Mueller coefficients $\mathbf{m}=\left[m_{00}, m_{01}, \ldots, m_{33}\right]^{T}$. Moreover, $\mathbf{P}$ is the $(50 \times 16)$ transformation matrix of the set-up and depends on the thickness configuration chosen for the plates. According to previous investigations about the propagation of random noise on Mueller matrices [11], the value of the term denoted by $E W V$ (Equally Weighted Variance) and linked to the variance $\operatorname{Var}(\mathbf{m})_{k}$ of the $m_{i j}$ components of $\mathbf{m}$ can be calculated from the singular values, $\mu_{k}$, of the transformation matrix, $\mathbf{P}$, through use of the 
relationship $E W V=\sum_{k=1}^{16} \operatorname{Var}(\mathbf{m})_{k}=\sum_{k=1}^{16} \frac{1}{\mu_{k}^{2}}$. For the single-channel-SMMP, this calculation in [11] led to $E W V=3524$. By comparison, in the case of the two-channel-SMMP, $E W V=1142$, which means a reduction of this parameter by about 3 . The two-channel-SMMP is, thus, about three times less disturbed by the experimental random noise on instantaneous measurements of Mueller matrices.

\section{4 - Systematic errors}

\section{A - Influence of the plates}

The calibration of an SMMP was described in [10], in the case where the thickness of the first plate, $e$, is used as reference. It showed that the quantification of the window phase, $\phi_{w}$, and those of the thickness errors on the other plates, $e_{2}, e_{3}$ and $e_{4}$, with respect to the ideal configuration $(e, e, 5 e, 5 e)$ are a must. These values are determined through measurements of two known media: for example, vacuum (when the sample is missing) and either a linear polarizer, or a half-wave retardation plate. One should be aware that, in experiments, inaccuracies in the determination of the values of $\phi_{w}, e_{2}, e_{3}$ and $e_{4}$ are unavoidable and lead to absolute errors denoted here as $\Delta \phi_{w}, \Delta e_{2}, \Delta e_{3}$ and $\Delta e_{4}$. Furthermore, the wave-plates in the set-up are ideally aligned in the configuration $\left(45^{\circ}, 0^{\circ}, 0^{\circ}, 45^{\circ}\right)$ depicted in Fig.1. Therefore, the absolute errors $\left(\Delta \alpha_{1}, \Delta \alpha_{2}, \Delta \alpha_{3}, \Delta \alpha_{4}\right)$ on the alignment angles of the elements yield an additional source of systematic errors. In this study, the impact of these errors on a measured Mueller matrix was investigated via simulations run on varying the values of $\Delta \phi_{w}, \Delta e_{l}(l=2,3,4)$ and $\Delta \alpha_{m}$ $(m=1,2,3,4)$ and on assuming that the element under test was a quarter-wave plate oriented at $120^{\circ}$. Figure 2 compares the effects generated by the systematic errors with the single-channel- 
SMMP and the two-channel-SMMP. It clearly shows a reduction of the impact by systematic errors with the latter. It is also worth noting that the two-channel-SMMP allows an overdetermination of the system with no additional source of systematic errors, e.g. another waveplate or a polarizer. Concerning the influence of the Wollaston prism, spatial filtering should guarantee to be in the angle tolerance of the element, and one can reasonably expect that, with a 10-nm broadband source used here, the system will be unaffected by the Wollaston-induced changes on the beam angle.

These promising results with the two-channel-SMMP simulations drove us to make measurements in order to assess the level of upgrade with this device. The experimental set-up was very similar to the above-described one, except that the Wollaston prism was replaced with an output linear polarizer (alike the one in the single-channel-SMMP device) set at first at $0^{\circ}$, and then at $90^{\circ}$. Moreover, a multimodal optical fiber was used to enter the detection system; however, its use with a coherent beam generates some interference between spatial modes, which depend on the injection angle, and are responsible for modulations in the shape of the spectrum, $s(\lambda)$. In order to avoid coherence effects and be certain that $s(\lambda)$ is alike for $I^{\prime \prime}(\lambda)$ and $I^{\perp}(\lambda)$ signals, the medium under study was imaged on a rotating depolarizing medium, in turn imaged on the fiber entrance. Moreover, the reconstruction of the Mueller matrix, normalized by $m_{00}$, involves a step of normalization of Eqs.(3) and (4) by a given reference. Consequently, differences in coupling efficiency between the fibers do not occur. Table 1 gives experimental Mueller matrices relative to the single-channel-SMMP and the two-channel-SMMP with their associated physical parameters (calculated from a Lu and Chipman decomposition [12]) for a commercial quarter-wave plate oriented at $120^{\circ}$ as a test medium. The experiment was driven with $10 \mu$ s acquisition time exposure and 100 accumulations so as to reduce random noise. 
Therefore, only systematic errors remain in the results. Table1 shows that the measurements made with the two-channel-SMMP are more precise than those by the single-channel-SMMP. Indeed, the improvement is particularly identifiable on the values of $P_{D}$ and $D$, which are closer to the ideal ones in the case of the two-channel-SMMP. In both cases, the parameter $R$ always stands in the acceptance values given by the manufacturer and the orientation of the fast axis $\alpha$ is in the error bar of the experimentation.

\section{$B$ - Influence of the source and the medium under study}

With the two-channel SMMP, an additional source of concern is about $s(\lambda)$ measurement. Indeed, in a classical Mueller polarimeter, the polarization states are sequentially generated, which means that intensity variations with time have to be taken into account. On the other hand, with a snapshot polarimeter, the polarization states are generated simultaneously owing to wavelength coding, and thus the intensity variations with wavelength have to be considered. The signal, $s(\lambda)$, has two origins: the shape of the source spectrum and the non-uniform spectral response of the medium under study (produced, for example, by multiple reflection-generated interferences). One should also note that both take place at the same time. The knowledge of the Fourier peak magnitudes is essential for the reconstruction of a Mueller matrix. Since $s(\lambda)$ is a term of a product in the measurement domain (Eqs.(3) and (4)), it acts as a convolution product in the Fourier domain, and thus it can be at the origin of systematic errors on the reconstructed Mueller matrix. Hence, $s(\lambda)$ must be calculated to take into account the correction (dividing $I^{\prime \prime}(\lambda)$ and $I^{\perp}(\lambda)$ by $\left.s(\lambda)\right)$ and make an accurate reconstruction. Summation of the two signals, $I^{\prime \prime}(\lambda)$ and $I^{\perp}(\lambda)$, from Eqs.(3) and (4) leads to:

$$
I^{\prime \prime}(\lambda)+I^{\perp}(\lambda)=s(\lambda) \cdot f(\lambda)
$$


where $f(\lambda)$ is given by:

$$
64 f(\lambda)=32 m_{00}+16 m_{02}+32 m_{01} \cos \left(f_{0} \lambda\right)-16 m_{02} \cos \left(2 f_{0} \lambda\right)-16 m_{03} \sin \left(2 f_{0} \lambda\right)
$$

\section{1- Non-absorbing medium}

A given medium is either non-absorbing or absorbing. If it is non-absorbing, the first row (with the exception of coefficient, $\left.m_{00}\right)$ of its Mueller matrix is null $\left(m_{01}=m_{02}=m_{03}=0\right)$. As a consequence, Eq.(5) becomes $I^{\prime \prime}(\lambda)+I^{\perp}(\lambda)=1 / 2 . s(\lambda) \cdot m_{00}$, which gives direct access to $s(\lambda)$. This step was experimentally performed in this study. The level of improvement was assessed through use of the value of the Frobenius norm, $\|\Delta M\|_{F}=\sqrt{\sum_{i j} \mid m_{i j} \text { exp }-\left.m_{i j}^{t h}\right|^{2}}$, which represents the global difference between the theoretical Mueller matrix and the experimental one. This value is generally used when dealing with experimental errors on Mueller matrices [13]. Figure 3(a) illustrates the variations of intensity versus $\lambda$, which are attributable to the nature of the source. For vacuum, calculations for $\|\Delta M\|_{F}$ gave values of 0.04 without source spectrum correction against 0.017 with it; this significant difference between values is the sign of improvement in the measurement accuracy. To investigate the influence of the interference signal given by a non-absorbing medium, a quartz wave-plate cut perpendicular to the optical axis and with no anti-reflection treatment of the faces was used as the sample. The $s(\lambda)$ spectrum associated to this plate is presented in Fig.3(b). For this plate, the value of $\|\Delta M\|_{F}$ with no correction is 0.155 against 0.05 with correction, thereby reducing the error by a factor of around three. 


\section{2- Absorbing medium}

In the case of an absorbing medium, the Mueller coefficients, $m_{01}, m_{02}$ and $m_{03}$, are different from 0 , and thus there is no way to directly determine $s(\lambda)$. However, $s(\lambda)$ can be retrieved by carrying out a pre-calibration procedure as follows: let us consider an unknown medium, whose Mueller matrix is $[M]$. A half-wave plate oriented at first at $0^{\circ}([B(\pi, 0)])$, and then at $45^{\circ}([B(\pi, \pi / 4)])$, as well as a polarizer at $0^{\circ}\left(\left[P_{o l}(0)\right]\right)$ are both set in front of this medium. Two artificial media, $\left[M_{1}\right]$ and $\left[M_{2}\right]$, are thus generated as follows:

$$
\begin{aligned}
& {\left[M_{1}\right]=[M]\left[P_{o l}(0)\right] \cdot[B(\pi, 0)]} \\
& {\left[M_{2}\right]=[M]\left[P_{o l}(0)\right] \cdot[B(\pi, \pi / 4)]}
\end{aligned}
$$

Four intensities are then measured: $I_{1}^{\prime \prime}(\lambda)$ and $I_{1}^{\perp}(\lambda)$ concern $\left[M_{l}\right]$, whereas $I_{2}^{\prime \prime}(\lambda)$ and $I_{2}^{\perp}(\lambda)$ concern $\left[M_{2}\right]$. The sum of $I_{1}^{\perp}(\lambda)+I_{2}^{\perp}(\lambda)$, or that of $I_{1}^{\prime \prime}(\lambda)+I_{2}^{\prime \prime}(\lambda)$, is equivalent to the measurement with a single-channel-SMMP of a medium represented by the Mueller matrix, $\left[M_{t o t}\right]=\left[M_{1}\right]+\left[M_{2}\right]$. By using Eqs.(7) and (8), one gets $\left[M_{t o t}\right]$ as follows:

$$
\left[M_{t o t}\right]=[M] \cdot\left(\begin{array}{cccc}
1 & 0 & 0 & 0 \\
1 & 0 & 0 & 0 \\
0 & 0 & 0 & 0 \\
0 & 0 & 0 & 0
\end{array}\right)=\left(\begin{array}{llll}
m_{00}+m_{01} & 0 & 0 & 0 \\
m_{10}+m_{11} & 0 & 0 & 0 \\
m_{20}+m_{21} & 0 & 0 & 0 \\
m_{30}+m_{31} & 0 & 0 & 0
\end{array}\right)
$$

The sum of the four intensities, $I_{1}^{\prime \prime}(\lambda)+I_{1}^{\perp}(\lambda)+I_{2}^{\prime \prime}(\lambda)+I_{2}^{\perp}(\lambda)$, is equivalent to the measurement of the medium, $\left[M_{t o t}\right]$, with a two-channel-SMMP:

$$
I_{t o t}=I_{1}^{\prime \prime}(\lambda)+I_{1}^{\perp}(\lambda)+I_{2}^{\prime \prime}(\lambda)+I_{2}^{\perp}(\lambda)=s(\lambda) \cdot f(\lambda)
$$

where $f(\lambda)$ is taken from the relation: $64 f(\lambda)=32 m_{00}+32 m_{01}$, which is easily identifiable thanks to Eq.(6). This means that $s(\lambda)$ is retrieved in the case of an absorbing medium. The experimental validation of this method was made on setting the previous quartz wave-plate in front of a linear 
polarizer at $30^{\circ}$ to generate the medium, $[M]$, which is thus absorbing. The two components of this medium (quartz wave-plate and linear polarizer) were characterized independently so as to generate the theoretical matrix from the product of their Mueller matrices. The value found for $\|\Delta M\|_{F}$ with no correction was 0.205 against 0.044 with correction, which means that further to the correction, the accuracy was about five times better than without it.

\section{5- Conclusion}

This study demonstrated the benefits of the proposed upgrade of a single-channel-SMMP. It, indeed, led to a noticeable reduction of the effects generated by both random noise and the systematic errors associated to inaccuracies on thicknesses and alignments of the retarder plates. Moreover, the use of this dual-measurement technique leads to an over-determined system, with no additional systematic errors. Furthermore, the dual-beam technique allows one to correct the effects by $s(\lambda)$ (issued from the source spectrum and interference generated by the medium under study). This correction can be made instantaneously for a non-absorbing medium. In the case of an absorbing medium with no evolution of $s(\lambda)$ over the experiment, a pre-calibration step is needed before performing snapshot measurements. Our experiments showed that the twochannel-SMMP proved to be more precise than a single-channel-SMMP.

\section{Acknowledgments}

The authors would like to thank M. G. Zion for his technical assistance and Dr M.P. Friocourt for her advice about writing in the English language. 


\section{References}

1. R. Ossikovski, E. Garcia-Caurel, and A. De Martino, "Product decompositions of experimentally determined non-depolarizing Mueller matrices", Phys. Stat. Sol.(c) 5, No. 5, $1059-1063(2008)$

2. C. Baravian, J. Dillet, F. Caton, and J.P. Decruppe, "Birefringence determination in turbid media,” Phys. Rev. E 75, 032501 (2007)

3. F.Boulvert, B.Boulbry, G.Le Brun, B.Le Jeune, S.Rivet, and J.Cariou, "Analysis of the depolarizing properties of irradiated pig skin,” J. opt. A, Pure Appl. Opt. 7, 21-28 (2005)

4. F. Delplancke, "Automated high-speed Mueller matrix scatterometer," Appl. Opt. 36, 5388$5395(1997)$

5. R. W. Collins and J. Koh, “"Dual rotating-compensator multichannel ellipsometer: instrument design for real-time Mueller matrix spectroscopy of surfaces and films," J. Opt. Soc. Am. A 16, 1997-2006 (1999)

6. E. Garcia-Caurel, A. De Martino, B. Drévillon, "Spectroscopic Mueller polarimeter based on liquid crystal devices", Thin Solid Films, 455-456, 120-123 (2004)

7. K. Oka and T. Kato, "Spectroscopic polarimetry with a channeled spectrum," Opt. Lett. 24, 1475-1477 (1999)

8. N. Hagen, K. Oka, and E. L. Dereniak, "Snapshot Mueller matrix spectropolarimeter," Opt. Lett. 32, 2100-2102 (2007)

9. M. Dubreuil, S. Rivet, B. Le Jeune, and J. Cariou, "Snapshot Mueller matrix polarimeter by wavelength polarization coding," Opt. Express 15, 13660-13668 (2007)

10. M. Dubreuil, S. Rivet, B. Le Jeune, and J. Cariou, "Systematic errors specific to a snapshot Mueller matrix polarimeter," Appl. Opt. 48, 1135-1142 (2009) 
11. P. Lemaillet, S. Rivet, and B. Le Jeune, "Optimization of a snapshot Mueller matrix polarimeter," Opt. Lett. 33, 144-146 (2008)

12. S-Y.Lu and R.A.Chipman, "Interpretation of Mueller matrices based on polar decomposition”, J. Opt. Soc. Am. A 13, 1106-1113 (1996)

13. F.Boulvert, G.Le Brun, B.Le Jeune, J.Cariou, L.Martin, "Decomposition algorithm of an experimental Mueller matrix”, Opt. Comm. 282, 692-704 (2009) 


\section{List of Figure captions}

Figure 1: Experimental set-up for a two-channel-SMMP in the $(e, e, 5 e, 5 e)$ configuration. A single-channel-SMMP gives access to only $I^{\perp}(\lambda)$.

Figure 2: Simulations of the impact by systematic errors in the cases of a single-channel-SMMP (continuous line) and a two-channel-SMMP (squares). The medium under study was a quarterwave plate oriented at $120^{\circ}$. The inaccuracy of the window phase, $\Delta \phi_{w}$, is expressed in radians. The set-up used in the simulations consisted of four calcite plates $(\Delta n=0.166)$ of thicknesses $e=2.08 \mathrm{~mm}$ for plates 1 and 2 , and $5 e=10.4 \mathrm{~mm}$ for plates 3 and 4 . The source had a broadband spectrum with $\lambda_{0}=829 \mathrm{~nm}$, and the analysis window of the detection system was $\Delta \lambda=10 \mathrm{~nm}$ sampled with 512 pixels. The thickness error inaccuracies, $\Delta e_{2}, \Delta e_{3}$ and $\Delta e_{4}$, are expressed in units of wavelengths, the misalignment errors, $\Delta \alpha_{1}, \Delta \alpha_{2}, \Delta \alpha_{3}$ and $\Delta \alpha_{4}$, are given in degrees. The term used to quantify the measurement errors is the Frobenius norm, $\|\Delta M\|_{F}=\sqrt{\sum_{i j}\left|m_{i j}^{\text {errors }}-m_{i j}^{i d e a l}\right|^{2}} ;$ it corresponds to the difference between the Mueller matrix with systematic errors $\left(m_{i j}^{\text {errors }}\right)$ and the ideal one $\left(m_{i j}^{i d e a l}\right)$. All of the $m_{i j}$ coefficients are normalized by $m_{00}$.

Figure 3: Experimental spectra: (a) vacuum (source shape) and (b) quartz wave plate (multiplereflection interference signal). 


\section{List of Table captions}

Table 1: Experimental Mueller matrices for a quarter-wave plate with its fast-axis oriented at $120^{\circ}$ with either the single-channel-SMMP (a) or the two-channel-SMMP (b). The parameters $P_{D}$ (depolarization index), $D$ (diattenuation), $R$ (retardance), $\varepsilon_{r}$ (ellipticity), $\alpha_{r}$ (orientation of the fast-axis) are calculated from a Lu and Chipman decomposition. The plate is a quartz wave plate (QWPO-830-10-4-R15 from Melles Griot) whose retardance is given by $\mathrm{R}=90^{\circ} \pm 1^{\circ}$ at $829 \mathrm{~nm}$ and ellipticity $\varepsilon_{\mathrm{r}}=0$. The plate is assumed to be non-depolarizing $\left(\mathrm{P}_{\mathrm{D}}=1.000\right)$ and nonabsorbing $(\mathrm{D}=0.000)$. The plate is aligned by a rotating mount at the position $\alpha_{\mathrm{r}}=120.00^{\circ} \pm 0.1^{\circ}$. All of the Mueller coefficients are normalized by $\mathrm{m}_{00}$. 
Figure 1: Experimental set-up for a two-channel-SMMP in the $(e, e, 5 e, 5 e)$ configuration. A single-channel-SMMP gives access to only $I^{\perp}(\lambda)$.

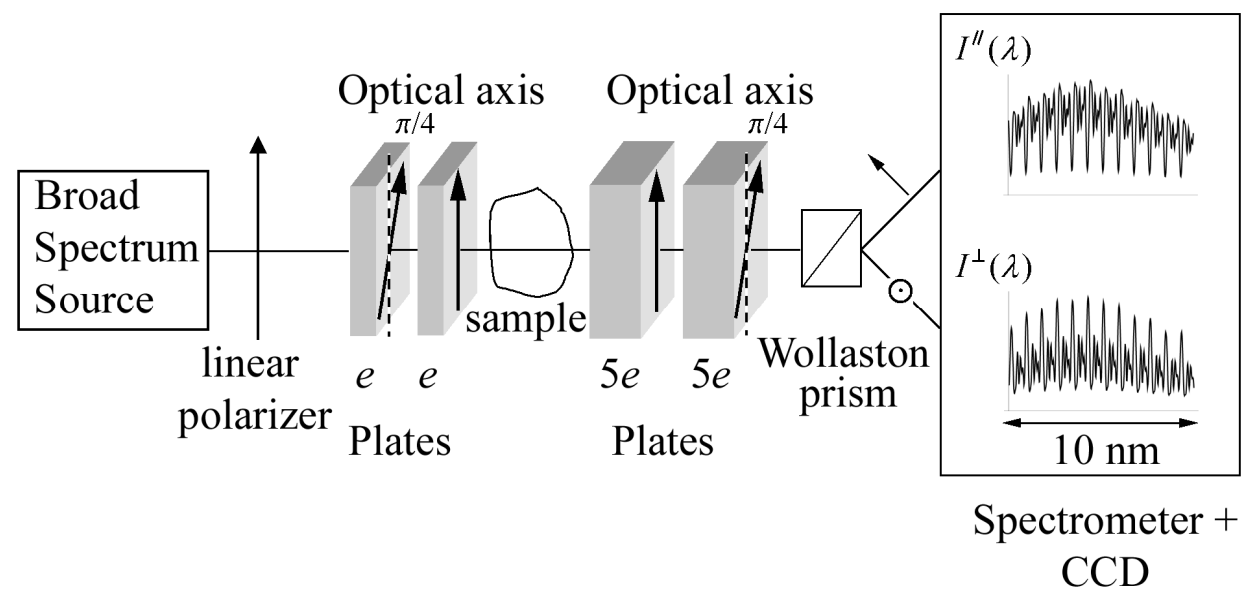


Figure 2: Simulations of the impact by systematic errors in the cases of a single-channel-SMMP (continuous line) and a two-channel-SMMP (squares). The medium under study was a quarterwave plate oriented at $120^{\circ}$. The inaccuracy of the window phase, $\Delta \phi_{w}$, is expressed in radians. The set-up used in the simulations consisted of four calcite plates $(\Delta n=0.166)$ of thicknesses $e=2.08 \mathrm{~mm}$ for plates 1 and 2 , and $5 e=10.4 \mathrm{~mm}$ for plates 3 and 4 . The source had a broadband spectrum with $\lambda_{0}=829 \mathrm{~nm}$, and the analysis window of the detection system was $\Delta \lambda=10 \mathrm{~nm}$ sampled with 512 pixels. The thickness error inaccuracies, $\Delta e_{2}, \Delta e_{3}$ and $\Delta e_{4}$, are expressed in units of wavelengths, the misalignment errors, $\Delta \alpha_{1}, \Delta \alpha_{2}, \Delta \alpha_{3}$ and $\Delta \alpha_{4}$, are given in degrees. The term used to quantify the measurement errors is the Frobenius norm, $\|\Delta M\|_{F}=\sqrt{\sum_{i j}\left|m_{i j}^{\text {errors }}-m_{i j}^{i d e a l}\right|^{2}}$; it corresponds to the difference between the Mueller matrix with systematic errors $\left(m_{i j}^{\text {errors }}\right)$ and the ideal one $\left(m_{i j}^{i d e a l}\right)$. All of the $m_{i j}$ coefficients are normalized by $m_{00}$.
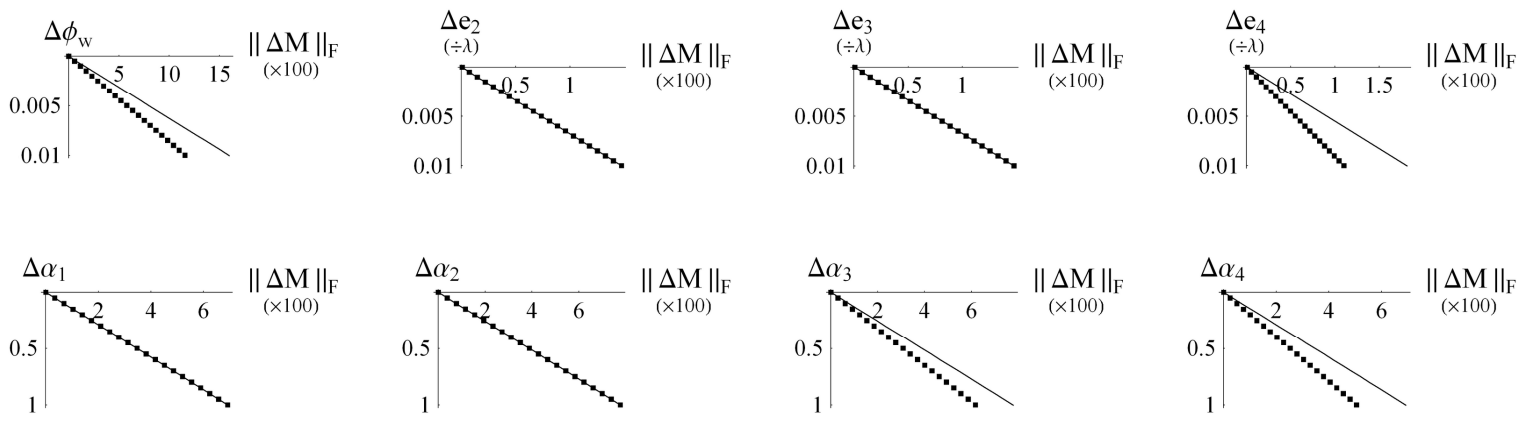
Figure 3: Experimental spectra: (a) vacuum (source shape) and (b) quartz wave plate (multiplereflection interference signal).

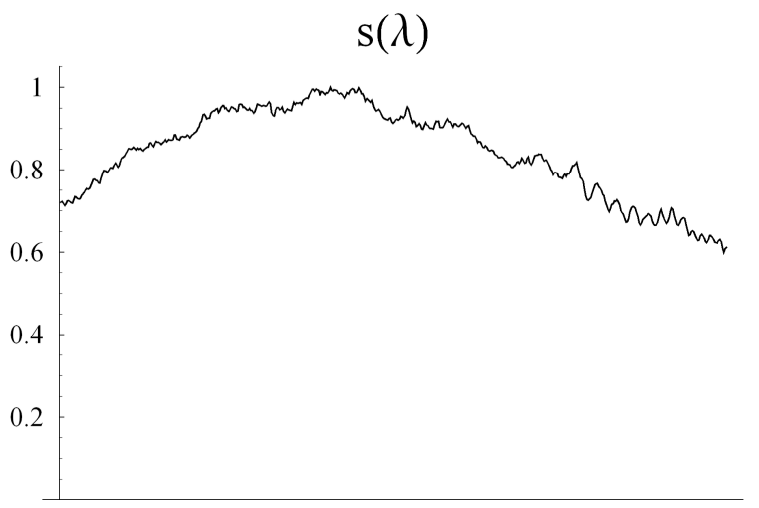

(a)

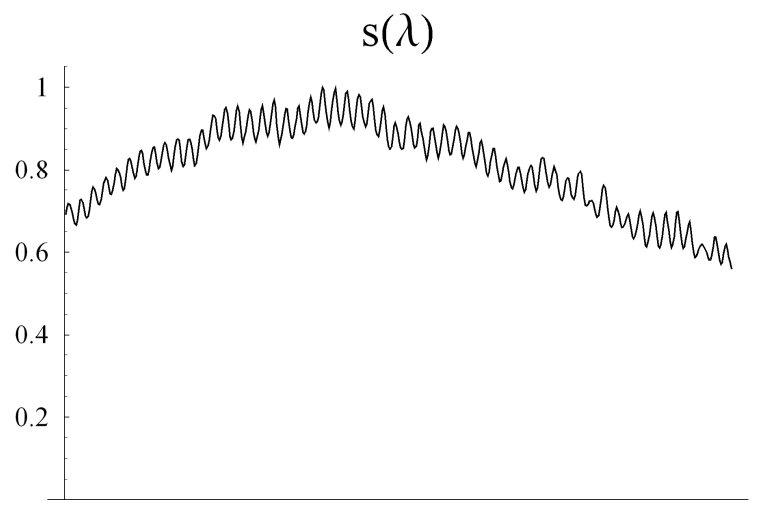

(b) 
Table 1: Experimental Mueller matrices for a quarter-wave plate with its fast-axis oriented at $120^{\circ}$ with either the single-channel-SMMP (a) or the two-channel-SMMP (b). The parameters $P_{D}$ (depolarization index), $D$ (diattenuation), $R$ (retardance), $\varepsilon_{r}$ (ellipticity), $\alpha_{r}$ (orientation of the fast-axis) are calculated from a Lu and Chipman decomposition. The plate is a quartz wave plate (QWPO-830-10-4-R15 from Melles Griot) whose retardance is given by $\mathrm{R}=90^{\circ} \pm 1^{\circ}$ at $829 \mathrm{~nm}$ and ellipticity $\varepsilon_{\mathrm{r}}=0$. The plate is assumed to be non-depolarizing $\left(\mathrm{P}_{\mathrm{D}}=1.000\right)$ and nonabsorbing $(\mathrm{D}=0.000)$. The plate is aligned by a rotating mount at the position $\alpha_{\mathrm{r}}=120.00^{\circ} \pm 0.1^{\circ}$. All of the Mueller coefficients are normalized by $\mathrm{m}_{00}$.

\section{(a)}

$$
\begin{gathered}
{\left[\begin{array}{cccc}
1.000 & -0.010 & 0.002 & 0.005 \\
0.007 & 0.259 & 0.444 & 0.865 \\
0.000 & 0.423 & 0.753 & -0.509 \\
-0.002 & -0.878 & 0.483 & 0.006
\end{array}\right]} \\
P_{D}=1.004, D=0.011 \\
R=89.63^{\circ}, \varepsilon_{r}=0.36^{\circ}, \alpha_{r}=120.05^{\circ}
\end{gathered}
$$

(b)

$$
\begin{gathered}
{\left[\begin{array}{cccc}
1.000 & 0.000 & 0.000 & 0.000 \\
0.006 & 0.258 & 0.445 & 0.864 \\
0.003 & 0.435 & 0.746 & -0.512 \\
0.001 & -0.868 & 0.485 & -0.002
\end{array}\right]} \\
\quad P_{D}=1.001, D=0.0002 \\
R=90.09^{\circ}, \varepsilon=0.25^{\circ}, \alpha_{r}=119.89^{\circ}
\end{gathered}
$$

